University of Windsor

Scholarship at UWindsor

3-15-2014

\title{
Coalition analysis with preference uncertainty in group decision support
}

\author{
Kevin Li \\ University of Windsor \\ Takehiro Inohara \\ Tokyo Institute of Technology \\ Haiyan Xu \\ Nanjing University of Aeronautics and Astronautics
}

Follow this and additional works at: https://scholar.uwindsor.ca/odettepub

Part of the Business Commons

\section{Recommended Citation}

$\mathrm{Li}$, Kevin; Inohara, Takehiro; and Xu, Haiyan. (2014). Coalition analysis with preference uncertainty in group decision support. Applied Mathematics and Computation, 231, 307-319.

https://scholar.uwindsor.ca/odettepub/93

This Article is brought to you for free and open access by the Odette School of Business at Scholarship at UWindsor. It has been accepted for inclusion in Odette School of Business Publications by an authorized administrator of Scholarship at UWindsor. For more information, please contact scholarship@uwindsor.ca. 


\title{
Coalition analysis with preference uncertainty in group decision support
}

\author{
Kevin W. $\mathrm{Li}^{1}$, Takehiro Inohara ${ }^{\star 2}$, and Haiyan $\mathrm{Xu}^{3}$ \\ ${ }^{1}$ Odette School of Business, University of Windsor, Windsor, Ontario, N9B 3P4. Canada \\ ${ }^{2}$ Department of Value and Decision Science, Tokyo Institute of Technology, W9-38, 2-12-1 \\ O-okayama Meguro, Tokyo 152-8552, Japan \\ ${ }^{3}$ College of Economics and Management, Nanjing University of Aeronautics and Astronautics, \\ Nanjing, Jiangsu, China
}

May 6, 2013

\begin{abstract}
Coalition analysis is extended to incorporate uncertain preference into three stability concepts, general metarationality (GMR), symmetric metarationality (SMR), and sequential stability (SEQ) under the paradigm of the graph model for conflict resolution. As a follow-up analysis in the graph model, coalition analysis aims to assess whether equilibriums under individual calculations are vulnerable to coalition moves and countermoves and, hence, become unstable under coalition stabilities. Coalition analysis has been considered for transitive graph models with simple preference under four stabilities, Nash, GMR, SMR, and SEQ, as well as general graph models with uncertain preference for the Nash stability. This paper introduces preference uncertainty into coalition stabilities under GMR, SMR, and SEQ for general graph models that can be transitive or intransitive. Depending on the focal coalition's different attitudes towards preference uncertainty, four different extensions are presented. Interrelationships of coalition stabilities are investigated within each
\end{abstract}

${ }^{*}$ Corresponding author. E-mail: inohara@valdes.titech.ac.jp (Takehiro Inohara). 
extension and across the four extensions. A case study is carried out to illustrate how to apply the proposed coalition stabilities.

Keywords: Conflict analysis, coalition analysis; preference uncertainty; graph model.

\section{Introduction}

Conflict and confrontation among agents with distinct interests may occur at many different settings and scales [3]. To handle strategic conflicts, different approaches have been put forward such as hypergame analysis [7], drama theory [2], and the graph model for conflict resolution [3]. As a simple but flexible group decision technology, the graph model is a proven and invaluable tool for modeling and analyzing strategic conflict in which two or more self-interested agents are in dispute over some issues $[3,10]$. When a conflict model is established within the graph model framework, two stages are involved: modeling and analysis. In the modeling stage, an analyst or stakeholder identifies two or more decision-makers (DMs) involved in the conflict situation, each DM's available courses of action or options, feasible states formed by all DMs' plausible option selections, state transitions among feasible states controlled by each DM, as well as all DMs' preference over feasible states [3]. Once a conflict model is set up, the analysis stage involves a standard stability analysis and some follow-up analyses such as coalition analysis $[8,9,11,22]$ and status quo analysis $[15,16,21]$. The stability analysis assesses stability of each state from each DM's perspective and a state that is stable for all DMs is called an equilibrium, corresponding to a potential resolution for the conflict model. The stability analysis is built upon a noncooperative concept with an underlying assumption that each DM acts independently for its own best interests after calculating its moves as well as coutermoves by its opponents. Following this line of thinking, the status quo analysis takes a forward looking perspective to assess how DMs act and react to direct a conflict from a status quo state or initial state to any particular equilibrium that is of interest to the analyst or stakeholders $[16,17,21]$. On the other hand, the other post-stability analysis, coalition analysis follows a cooperative viewpoint and assesses whether individual DMs can jointly 
improve their position by joining a coalition [8,11,22].

Coalition formation and stability have been an active research area in game theory $[1,12,13,19]$. The coalition analysis considered here is confined to the graph model for conflict resolution paradigm. As Kilgour et al. [11] put it, coalition analysis assesses whether self-interested and independent DMs can gain by forming a coalition and coordinating their moves. This paper follows the idea in $[11,22]$ and treats coalition analysis as a post-stability analysis. The implication is that only equilibria identified in the stability analysis stage will be examined for coalition stability. The rationale is that a non-equilibrium state is not sustainable as at least one DM is expected to deviate from it unilaterally based on the DM's calculations. An equilibrium, on the other hand, is expected to sustain for a while since no DM is motivated to depart from it as per individual contemplations. However, when two or more DMs form a coalition, an equilibrium may be upset via a sequence of joint moves by the coalition members. In this case, the target state should also be an equilibrium as any non-equilibrium state is transient. This process is referred to as an "equilibrium jump" in [11]. Understandably the target state of an equilibrium jump should presumably make all members in the coalition better off and cannot be achieved by any DM acting individually. Coalition analysis, therefore, aims to alert the analyst whether such a coalition exists and, if existent, which equilibria are vulnerable to equilibrium jumps and how these jumps are attained by coalition joint moves.

When a state is assessed for individual stability, different solution concepts such as Nash stability (Nash) [18], general metarationality (GMR) [7], symmetric metarationality (SMR) [7], and sequential stability (SEQ) have been proposed to characterize DMs' distinct behavioural patterns in face of conflict [3]. For details of the characteristics and interrelationships of these solution concepts, readers are referred to Fang et al. [3] and the original references therein.

The original graph model methodology employs a simple preference structure, consisting of strict preference $(>)$ and indifference $(\sim)$ relations, to characterize DMs' relative preference over feasible outcomes. To accommodate the case that some preference infor- 
mation is unknown to the analyst, Li et al. [14] develop a non-probabilistic framework to handle preference uncertainty in the graph model where a new binary relation $U$ is introduced to represent a DM's uncertainty about its preference between two states. The four solution concepts, Nash, GMR, SMR, and SEQ, have been redefined based on the extended preference structure. Depending on how unknown preferences are incorporated, four versions of stability definitions are put forward and labeled as a, b, c, and d accordingly. These different extensions are conceived to reflect the focal DM's distinct attitudes towards preference uncertainty, ranging from conservative, to mixed and aggressive [14].

Within the graph model framework, coalition analysis has been actively studied. Motivated by the strong equilibrium concept by Aumann [1], Kilgour et al. [11] introduce a coalition Nash stability concept with simple preference and the aim is to alert whether a status quo equilibrium can be upset by joint moves coordinated by a subset of DMs or a coalition. Subsequently, Inohara and Hipel [8] extend the idea and define coalition GMR, SMR, and SEQ stability. The interrelationships of these coalition stabilities are then examined [9]. For tractability, the aforesaid research has been confined to transitive graphs with the simple preference structure, in which consecutive moves by the same DM are allowed. By exploiting a convenient matrix system, Xu et al. [22] investigate coalition Nash stability with preference uncertainty for general graph models, where the requirement of no successive moves by the same DM is honoured to keep the new development consistent with the general decision rule in the graph model methodology. According to how uncertain preferences are incorporated, conservative and aggressive coalition Nash stabilities are introduced [22].

Building upon the research by Xu et al. [22] and Inohara and Hipel [8,9], the contribution of this article is to integrate preference uncertainty into coalition GMR, SMR, and SEQ stabilities. To keep notation consistent with individual stabilities in Li et al. [14], four different versions of each coalition stability will be defined accordingly.

To illustrate how this new development can be applied in practice, a coalition analysis is conducted for a case study of bulk-water export conflict occurred in the Province of Newfoundland and Labrador in Canada. This conflict was first examined by Fang et al. [4] 
and a three-DM graph model is established to investigate strategic interactions among different stakeholders. Subsequently, preference uncertainty is introduced into the model to characterize the oscillating attitude of the provincial government towards bulk-water export from its jurisdiction [14]. These analyses furnish useful strategic advice on how different stakeholders may act and react to bring the conflict to potential resolutions. The current analysis moves one step further by investigating which equilibria are sustainable and will not be upset by coalition moves and which equilibria are likely to be transient and susceptible to be overturned by a subgroup of DMs coordinating their moves. The aim is to shed additional structural insights on whether any DM may further improve its position by joining a coalition.

To make the paper self-contained, the next section briefly reviews the graph model for conflict resolution and puts the current research in a proper context. Section 3 defines coalition GMR, SMR, and SEQ stabilities with preference uncertainty. Section 4 investigates interrelationships of coalition stabilities within each extension and across the four extensions, followed by an illustrative case study in Section 5. The paper concludes with some remarks in Section 6.

\section{Preliminaries}

\subsection{The graph model for conflict resolution}

A graph model consists of a set of DMs $N, 2 \leq|N|<\infty$, a finite set of feasible states $S$, a collection of digraphs $G_{i}=\left(S, A_{i}\right), i \in N$, where $S$ is the vertex set and $A_{i}$ is DM $i$ 's set of directed arcs in $G_{i}$ and depicts the moves among feasible states controlled by DM $i$, and relative preference over the feasible states for all DMs [3]. When a graph model is not too big, it is often convenient to draw an integrated digraph for all DMs where arcs are appropriately labeled with controlling DMs. As the graph model has the flexibility in characterizing common moves, it is possible that two arcs $a_{1}$ and $a_{2}$ may share the same pair of starting and terminal states $s_{1}$ and $s_{2}$, i.e., $a_{1}=\left(s_{1}, s_{2}\right)$ and $a_{2}=\left(s_{1}, s_{2}\right)$. In this case, these common moves must be controlled by different DMs such 
that $a_{1} \in A_{i}, a_{2} \in A_{j}, i, j \in N, i \neq j$.

Preference information plays a central role in conducting a stability analysis. The original graph model adopts a pair of binary relations $\left\{>_{i}, \sim_{i}\right\}$, the so-called simple preference, to represent DMs' relative preference over feasible states. In practice, the analyst may have a hard time in obtaining accurate preference information about the conflict when complicated and multiple criteria have to be evaluated $[5,6]$, and sometimes, even the DMs themselves cannot tell their true preference when the conflict is still ongoing and evolving. To handle partially unknown preferences, Li et al. [14] extend the simple preference structure to a triplet of binary relations $\left\{>_{i}, \sim_{i}, U_{i}\right\}$. For DM $i$ and any two states $s_{1}, s_{2} \in S, s_{1} U_{i} s_{2}$ indicates that DM $i$ is uncertain about its preference between $s_{1}$ and $s_{2}$, which may turn out to be either $s_{1}>_{i} s_{2}, s_{1} \sim_{i} s_{2}$ or $s_{2}>_{i} s_{1}$ when additional information becomes available.

For a general preference relation $P$ and three states $s_{1}, s_{2}$, and $s_{3}$, if $s_{1} P s_{2}$ and $s_{2} P s_{3}$ imply $s_{1} P s_{3}$, then $P$ is called transitive. The graph model does not require DMs' preferences to be transitive. As indicated in the sustainable development game in Li et al. [14], the uncertain preference relation can be intransitive. The new development here inherits this fine property and is applicable to both transitive and intransitive preferences.

\subsection{Existing coalition analysis in the graph model}

Coalition analysis has been studied in the graph model with simple preference under four solution concepts, Nash, GMR, SMR, and SEQ [8,9,11]. Xu et al. [22] further extend coalition Nash stability to accommodate uncertain preference. Below, a brief introduction is presented for the existing coalition analysis research.

A subset of DMs $H \subseteq N$ is called a coalition. Generally speaking, an empty coalition $H$ does not have any realistic meaning and, hence, it is hereafter assumed that $|H| \geq 1$. When $|H|=1$, the coalition $H$ is called trivial as it contains only a single DM. If $|H|>1$, the coalition is nontrivial.

Before stability definitions are introduced, it is necessary to characterize a DM's potential moves starting from a status quo state $s$, the following lists define DM $i$ 's 
possible moves incorporating different preference information $[3,14]$.

i) Reachable list: $R_{i}(s)=\left\{t \in S:(s, t) \in A_{i}\right\}$, describing the states that are reachable by DM $i$ unilaterally in one step.

ii) Unilateral improvement (UI) list: $R_{i}^{+}(s)=\left\{t \in S:(s, t) \in A_{i}\right.$ and $\left.t>_{i} s\right\}$, depicting the states that are attainable by DM $i$ unilaterally in one step and the terminal state is more preferred by the DM.

iii) Unilateral improvement or uncertain move (UIUM) list: $R_{i}^{+, U}(s)=\left\{t \in S:(s, t) \in A_{i}\right.$ and $t>_{i} s$ or $t U_{i} s$, defining the states that are achievable by DM $i$ unilaterally in one step and the DM either prefers the terminal state or is uncertain about its preference between the terminal and starting states.

For a general $n$-DM graph model $(|N|=n \geq 3)$, the opponents of a focal DM $i$ consist of a coalition $N-i$ and $|N-i| \geq 2$. When a focal DM contemplates a potential move away from a status quo, some solution concepts such as GMR, SMR, and SEQ require it to account for its opponents' countermoves. The countermoves by a nontrivial coalition is comprised of all states that are attainable from a particular state via a legal sequence of unilateral moves (UMs) by any subset of DMs in the coalition, where a DM may move more than once but not consecutively [3]. In existing coalition analysis research with simple preference in the graph model $[8,9,11]$, the rule of no consecutive moves by the same DM is lifted for the sake of tractability. The implication is that the applicability of the research is restricted to transitive graph models. Xu et al. [22] bring this restriction back to their coalition Nash stability with uncertain preference.

In parallel to $R_{i}(s)$ by a single $\mathrm{DM}$, the reachable list by a coalition $H \subseteq N$, denoted by $R_{H}(s)$, can be defined inductively below. If $s_{1} \in R_{H}(s)$, denote by $\Omega_{H}\left(s, s_{1}\right)$ the set of all last DMs in legal sequences of UMs from $s$ to $s_{1}$.

Definition 1 A unilateral move by $H$ is a member of $R_{H}(s) \subseteq S$, defined inductively by (1) if $j \in H$ and $s_{1} \in R_{j}(s)$, then $s_{1} \in R_{H}(s)$ and $j \in \Omega_{H}\left(s, s_{1}\right)$;

(2) if $s_{1} \in R_{H}(s), j \in H$ and $s_{2} \in R_{j}\left(s_{1}\right)$, then $s_{2} \in R_{H}(s)$ provided that $\Omega_{H}\left(s, s_{1}\right) \neq\{j\}$, and $j \in \Omega_{H}\left(s, s_{2}\right)$. 
Definition 1 ensures that a DM may move more than once in a legal sequence of moves by coalition $H$, but not in succession. If each move in the sequence is restricted to be a UIUM for the mover, one can have the following definition of coalition UIUM list $R_{H}^{+, U}(s)$. Similarly, if $s_{1} \in R_{H}^{+, U}(s)$, denote by $\Omega_{H}^{+, U}\left(s, s_{1}\right)$ the set of all last DMs in legal sequences of UIUMs from $s$ to $s_{1}$.

Definition 2 A unilateral improvement or uncertain move (UIUM) by $H$ is a member of $R_{H}^{+, U}(s) \subseteq S$, defined inductively by

(1) if $j \in H$ and $s_{1} \in R_{j}^{+, U}(s)$, then $s_{1} \in R_{H}^{+, U}(s)$ and $j \in \Omega_{H}^{+, U}\left(s, s_{1}\right)$;

(2) if $s_{1} \in R_{H}^{+, U}(s), j \in H$ and $s_{2} \in R_{j}^{+, U}\left(s_{1}\right)$, then $s_{2} \in R_{H}^{+, U}(s)$ provided that $\Omega_{H}^{+, U}\left(s, s_{1}\right) \neq$ $\{j\}$, and $j \in \Omega_{H}^{+, U}\left(s, s_{2}\right)$.

If each move in the legal sequence is restricted to be a UI for the mover, the resulting set will be the coalition UI list $R_{H}^{+}(s)$ starting from state $s$ for coalition $H$. For brevity, the definition of $R_{H}^{+}(s)$ is omitted here.

To define coalition stability, Kilgour et al. [11] introduce the following concept of coalition improvement (CI).

Definition 3 For a status quo state $s$ and a nonempty coalition $H \subseteq N$, a state $s_{1} \in$ $R_{H}(s)$ is a coalition improvement for $H$ from $s$, denoted by $s_{1} \in C R_{H}^{+}(s)$, iff $s_{1}>_{i} s$ for every $i \in H$.

It is worth noting that $C R_{H}^{+}(s) \neq R_{H}^{+}(s)$ as $R_{H}^{+}(s)$ denotes all states that are attainable by coalition $H$ via legal sequences of UIs from $s$. Although each individual move is a UI for the mover, there is no guarantee that the terminal state is preferred to $s$ by any DM involved in the sequence of moves [3]. On the contrary, $C R_{H}^{+}(s)$ ensures that the terminal state is always preferred to $s$ by all DMs in the coalition though an individual move along the sequence may not be a UI for the mover [11].

This paper envisages coalition analysis as a post-stability analysis and examines whether equilibria that are stable for all DMs under individual calculations are vulnerable to joint moves by coalitions. Therefore, both the status quo and target states 
are presumably equilibria. Next, coalition stabilities under Nash, GMR, SMR, and SEQ with simple preference are furnished.

Definition 4 State $s \in S$ is Nash stable for coalition $H \subseteq N$, denoted by $s \in S_{H}^{C N a s h}$, iff $C R_{H}^{+}(s)=\varnothing$.

This coalition Nash stability is adapted from [11], but $C R_{H}^{+}(s)$ here is expected to honour the rule of no successive moves by the same DM and, hence, this definition is applicable to both transitive and intransitive graph models. As mentioned earlier, an empty coalition does not have any realistic meaning, it is assumed hereafter that $|H|>0$. If $|H|=1$, then $H=\{i\}$ and $C R_{H}^{+}(s)=R_{i}^{+}(s)$. In this special case, Definition 4 is reduced to individual Nash stability [18]. However, for a nontrivial coalition $H \subseteq N,|H| \geq$ 2, coalition Nash stability checks the coalition improvement list $C R_{H}^{+}(s)$ rather than coalition members' individual UI lists $R_{i}^{+}(s), i \in H$.

If state $s \in S$ is Nash stable for every nonempty coalition $H \subseteq N$, it is called coalitionally Nash stable and denoted by $s \in S^{C N a s h}$.

For notational convenience, let $s \geq_{i} t$ represent $s>_{i} t$ or $s \sim_{i} t$, and $\Phi_{H}^{\leq}(s)=\{t \in S$ : $s \geq_{i} t$ for at least one $\left.i \in H\right\}$. It is apparent that $\Phi_{H}^{\leq}(s)$ considers only the preference relative to state $s$ without examining the reachability of those states from $s$ by $H$.

Definition 5 State $s \in S$ is general metarational (GMR) for coalition $H \subseteq N$, denoted by $s \in S_{H}^{C G M R}$, iff for every $s_{1} \in C R_{H}^{+}(s)$, there exists $s_{2} \in R_{N-H}\left(s_{1}\right)$ such that $s_{2} \in \Phi_{H}^{\leq}(s)$.

This coalition GMR definition is adapted from [8,9]. Instead of using subclass reachable lists as in $[8,9]$, a coalition reachable list by the opponents is adopted here. Under the assumption of no consecutive moves by the same DM in a legal sequence of UMs, Definition 5 is equivalent to Definition 7 in [8]. Similarly, if $H=\{i\}$, this definition is reduced to individual GMR [7].

If state $s \in S$ is GMR for every coalition $H \subseteq N$, it is called coalitionally GMR stable and denoted by $s \in S^{C G M R}$. 
Definition 6 State $s \in S$ is symmetric metarational ( $S M R$ ) for coalition $H \subseteq N$, denoted by $s \in S_{H}^{C S M R}$, iff for every $s_{1} \in C R_{H}^{+}(s)$, there exists $s_{2} \in R_{N-H}\left(s_{1}\right)$ such that $s_{2} \in \Phi_{H}^{\leq}(s)$ and $s_{3} \in \Phi_{H}^{\leq}(s)$ for all $s_{3} \in R_{H}\left(s_{2}\right)$.

This coalition SMR definition is adapted from $[8,9]$ and is equivalent to Definition 9 in [8]. The only difference here is, once again, the incorporation of no consecutive moves by the same DM in any sequence of UMs. Similarly, if $H=\{i\}$, Definition 6 is reduced to individual SMR [7].

If state $s \in S$ is SMR for every coalition $H \subseteq N$, it is called coalitionally SMR stable and denoted by $s \in S^{C S M R}$.

Definition 7 State $s \in S$ is sequentially stable ( $S E Q$ ) for coalition $H \subseteq N$, denoted by $s \in S_{H}^{C S E Q}$, iff for every $s_{1} \in C R_{H}^{+}(s)$, there exists $s_{2} \in R_{N-H}^{+}\left(s_{1}\right)$ such that $s_{2} \in \Phi_{H}^{\leq}(s)$.

Remark: By employing the subclass improvement list concept, the coalition SEQ stability definition introduced by Inohara and Hipel $[8,9]$ is able to consider credible sanctions by subcoalitions in the opponent camp. But this thoroughness comes at a significant computational cost as the number of subcoalitions increases exponentially with the number of opponents, making the calculation of subclass improvement list prohibitively difficult when the number of DMs in the model is large. In addition, it remains open about how to enforce the legality of sequences of moves by subcoalitions. As a tradeoff, it is proposed here to treat opponents $N-H$ as individuals. For transitive graph models, if a state is SEQ for a coalition under Definition 7 here, it is automatically SEQ for the coalition under Definition 11 in [8]. However, the inverse is generally not true. Similarly, when $H=\{i\}$, coalition SEQ would be reduced to individual SEQ stability.

If state $s \in S$ is SEQ for every coalition $H \subseteq N$, it is called coalitionally SEQ stable and denoted by $s \in S^{C S E Q}$.

$\mathrm{Xu}$ et al. [22] extend coalition Nash stability proposed by Kilgour et al. [11] by including uncertain preference in the coalition Nash stability definitions. Depending on the focal coalition's attitude towards risks associated with uncertain moves [14], aggressive and conservative coalition Nash stabilities are introduced [22]. As a preparation, a coalition improvement or uncertain move (CIUM) is defined first. 
Definition 8 For a status quo state $s$ and a nonempty coalition $H \subseteq N$, a state $s_{1} \epsilon$ $R_{H}(s)$ is a coalition improvement or uncertain move for $H$ from $s$, denoted by $s_{1} \in C R_{H}^{+, U}(s)$, iff $s_{1}>_{i} s$ or $s_{1} U_{i} s$ for every $i \in H$.

$C R_{H}^{+, U}(s)$ here differs from $R_{H}^{+, U}(s)$ in Definition 2 in that $R_{H}^{+, U}(s)$ cares about the process without worrying about the final result, but $C R_{H}^{+, U}(s)$ is concerned with the final result instead of the process. In other words, $R_{H}^{+, U}(s)$ requires each move in a legal sequence has to be a UIUM for the mover, but the preference relation between a final state and status quo for the coalition is not a concern at all. On the contrary, $C R_{H}^{+, U}(s)$ ensures that all coalition members prefer the terminal state to the status quo or are uncertain about their preference between these two states without examining the relative preference for each individual move along the legal sequence. Next, definitions of conservative and aggressive coalition Nash stability can be introduced [22]. To be consistent with the four extension notation in Li et al. [14], these two definitions are relabeled accordingly.

Definition 9 State $s \in S$ is aggressively Nash stable for coalition $H \subseteq N$, denoted by $s \in S_{H}^{C N a s h_{a}}$, iff $C R_{H}^{+, U}(s)=\varnothing$.

Definition 10 State $s \in S$ is conservatively Nash stable for coalition $H \subseteq N$, denoted by $s \in S_{H}^{C N a h_{b}}$, iff $C R_{H}^{+}(s)=\varnothing$.

Similar to the equilibrium concept in stability analysis under individual calculations, coalition Nash stability for aggressive and conservative DMs can be ascertained as follows: If state $s \in S$ is aggressively (or conservatively) Nash stable for every nonempty coalition $H \subseteq N$, it is called coalitionally aggressively (or conservatively) Nash stable and denoted by $s \in S^{C N a s h_{a}}\left(\right.$ or $s \in S^{C N a s h_{b}}$ ).

Although Definition 10 looks the same as Definition 4, they are different in the sense that Definition 10 is defined with preference uncertainty where uncertain moves are not strong enough motivation for the focal coalition to deviate from its status quo state. On the other hand, Definition 4 is defined for transitive graph models with simple preference and does not consider uncertain preference in its conception. As Nash stability does not examine countermoves by the opponents, similar to individual stability case in [14], 
$S_{H}^{C N a s h_{a}}=S_{H}^{C N a s h_{c}}$ and $S_{H}^{C N a s h_{b}}=S_{H}^{C N a s h_{d}}$. For brevity, coalition Nash stability under extensions c and d, which are identical to that under extensions a and b, respectively, will not be repeated here.

\section{Coalition stability with preference uncertainty in the graph model}

Coalition Nash stability has been extended to general graph models with uncertain preference by $\mathrm{Xu}$ et al. [22]. This section will consider coalition GMR, SMR, and SEQ stabilities with preference uncertainty.

DMs may exhibit different attitudes towards uncertainty when making choices. For instance, an optimistic DM tends to view uncertainty as a potential opportunity while a pessimistic DM may often regard an uncertain outcome as a risk. In addition, a DM's attitude towards uncertainty change with its status quo state: a DM who has little to lose is more likely to take an aggressive attitude towards uncertainty and treat it as a potential gain. On the contrary, a DM who has little to gain is highly likely to regard uncertain outcomes as a risk and adopt a conservative stance. To accommodate different attitudes towards preference uncertainty, Li et al. [14] define individual Nash, GMR, SMR, and SEQ stabilities with preference uncertainty under four forms, a, b, c, and d. The purpose of these four extensions is to characterize a focal DM with diverse attitudes towards preference uncertainty, ranging from aggressive, to mixed and conservative. When coalition GMR, SMR, and SEQ stability definitions are extended from graph models with simple preference [8] to those with uncertain preference, these four extensions are maintained depending on the focal coalition's attitude towards preference uncertainty.

Definition 11 State $s \in S$ is GMR for coalition $H \subseteq N$, denoted by $s \in S_{H}^{C G M R_{a}}$, iff for every $s_{1} \in C R_{H}^{+, U}(s)$, there exists $s_{2} \in R_{N-H}\left(s_{1}\right)$ such that $s_{2} \in \Phi_{H}^{\leq}(s)$.

Definition 12 State $s \in S$ is SMR for coalition $H \subseteq N$, denoted by $s \in S_{H}^{C S M R_{a}}$, iff for every $s_{1} \in C R_{H}^{+, U}(s)$, there exists $s_{2} \in R_{N-H}\left(s_{1}\right)$ such that $s_{2} \in \Phi_{H}^{\leq}(s)$ and $s_{3} \in \Phi_{H}^{\leq}(s)$ for all $s_{3} \in R_{H}\left(s_{2}\right)$. 
Definition 13 State $s \in S$ is $S E Q$ for coalition $H \subseteq N$, denoted by $s \in S_{H}^{C S E Q_{a}}$, iff for every $s_{1} \in C R_{H}^{+, U}(s)$, there exists $s_{2} \in R_{N-H}^{+, U}\left(s_{1}\right)$ such that $s_{2} \in \Phi_{H}^{\leq}(s)$.

In extension a, the focal coalition members are conceived to be aggressive as they are willing to deviate from the status quo state for uncertain outcomes (uncertainty is allowed at the incentive end for the focal coalition). While assessing sanctions from their opponents, at least one coalition member must be ascertained for a no-better-off position in order to successfully block the focal coalition (uncertainty is not allowed at the sanction end for the focal coalition).

Definition 14 State $s \in S$ is GMR for coalition $H \subseteq N$, denoted by $s \in S_{H}^{C G M R_{b}}$, iff for every $s_{1} \in C R_{H}^{+}(s)$, there exists $s_{2} \in R_{N-H}\left(s_{1}\right)$ such that $s_{2} \in \Phi_{H}^{\leq}(s)$.

Definition 15 State $s \in S$ is SMR for coalition $H \subseteq N$, denoted by $s \in S_{H}^{C S M R_{b}}$, iff for every $s_{1} \in C R_{H}^{+}(s)$, there exists $s_{2} \in R_{N-H}\left(s_{1}\right)$ such that $s_{2} \in \Phi_{H}^{\leq}(s)$ and $s_{3} \in \Phi_{H}^{\leq}(s)$ for all $s_{3} \in R_{H}\left(s_{2}\right)$.

Definition 16 State $s \in S$ is $S E Q$ for coalition $H \subseteq N$, denoted by $s \in S_{H}^{C S E Q_{b}}$, iff for every $s_{1} \in C R_{H}^{+}(s)$, there exists $s_{2} \in R_{N-H}^{+, U}\left(s_{1}\right)$ such that $s_{2} \in \Phi_{H}^{\leq}(s)$.

Compared to the coalition stability definitions in extension a, the only difference is that this extension does not treat uncertain moves as sufficient incentive for the focal coalition to deviate from the status quo. The focal coalition under this extension presumably exhibits mixed attitude towards preference uncertainty, conservative at the incentive end but aggressive at the sanction end [14]. Although Definitions 14, 15, and 16, respectively, look the same as Definitions 5, 6, and 7, they are in fact different in the sense that Definitions 14, 15, and 16 are conceived with preference uncertainty but uncertain moves are neither strong enough motivation for the focal coalition to deviate from the status quo nor allowed as valid sanctions to deter the focal coalition. On the other hand, Definitions 5, 6, and 7 are designed for graph models with simple preference.

For convenience, let $\Phi_{H}^{\leq, U}(s)=\left\{t \in S: s \geq_{i} t\right.$ or $s U_{i} t$ for at least one $\left.i \in H\right\}$. 
Definition 17 State $s \in S$ is GMR for coalition $H \subseteq N$, denoted by $s \in S_{H}^{C G M R_{c}}$, iff for every $s_{1} \in C R_{H}^{+, U}(s)$, there exists $s_{2} \in R_{N-H}\left(s_{1}\right)$ such that $s_{2} \in \Phi_{H}^{\leq, U}(s)$.

Definition 18 State $s \in S$ is SMR for coalition $H \subseteq N$, denoted by $s \in S_{H}^{C S M R_{c}}$, iff for every $s_{1} \in C R_{H}^{+, U}(s)$, there exists $s_{2} \in R_{N-H}\left(s_{1}\right)$ such that $s_{2} \in \Phi_{H}^{\leq, U}(s)$ and $s_{3} \in \Phi_{H}^{\leq, U}(s)$ for all $s_{3} \in R_{H}\left(s_{2}\right)$.

Definition 19 State $s \in S$ is $S E Q$ for coalition $H \subseteq N$, denoted by $s \in S_{H}^{C S E Q_{c}}$, iff for every $s_{1} \in C R_{H}^{+, U}(s)$, there exists $s_{2} \in R_{N-H}^{+, U}\left(s_{1}\right)$ such that $s_{2} \in \Phi_{H}^{\leq, U}(s)$.

Extension c assumes that uncertain moves are allowed as sufficient incentives and sanctions for the focal coalition and is designed to characterize focal coalition members with mixed attitude towards preference uncertainty: aggressive at the incentive end but conservative at the sanction end.

Definition 20 State $s \in S$ is GMR for coalition $H \subseteq N$, denoted by $s \in S_{H}^{C G M R_{d}}$, iff for every $s_{1} \in C R_{H}^{+}(s)$, there exists $s_{2} \in R_{N-H}\left(s_{1}\right)$ such that $s_{2} \in \Phi_{H}^{\leq, U}(s)$.

Definition 21 State $s \in S$ is SMR for coalition $H \subseteq N$, denoted by $s \in S_{H}^{C S M R_{d}}$, iff for every $s_{1} \in C R_{H}^{+}(s)$, there exists $s_{2} \in R_{N-H}\left(s_{1}\right)$ such that $s_{2} \in \Phi_{H}^{\leq, U}(s)$ and $s_{3} \in \Phi_{H}^{\leq, U}(s)$ for all $s_{3} \in R_{H}\left(s_{2}\right)$.

Definition 22 State $s \in S$ is $S E Q$ for coalition $H \subseteq N$, denoted by $s \in S_{H}^{C S E Q_{d}}$, iff for every $s_{1} \in C R_{H}^{+}(s)$, there exists $s_{2} \in R_{N-H}^{+, U}\left(s_{1}\right)$ such that $s_{2} \in \Phi_{H}^{\leq, U}(s)$.

Coalition stability definitions in extension d are devised for conservative focal coalitions: When contemplating incentives, they do not envision uncertain moves as opportunities (preference uncertainty is not allowed as incentives); while assessing sanctions, these DMs would view uncertain moves as potential harms (preference uncertainty is allowed as valid sanctions).

Similarly, if state $s \in S$ is GMR, SMR, or SEQ stable for each coalition $H \subseteq N$ under a particular extension, it is called coalitionally GMR, SMR, or SEQ stable under this extension, and denoted by $s \in S^{C G M R_{k}}, s \in S^{C S M R_{k}}$, or $s \in S^{C S E Q_{k}}, k=\mathrm{a}, \mathrm{b}, \mathrm{c}$, d. It is obvious that $S^{C G M R_{k}}=\cap_{H \subseteq N} S_{H}^{C G M R_{k}}, S^{C S M R_{k}}=\cap_{H \subseteq N} S_{H}^{C S M R_{k}}$, and $S^{C S E Q_{k}}=$ $\cap_{H \subseteq N} S_{H}^{C S E Q_{k}}, k=\mathrm{a}, \mathrm{b}, \mathrm{c}, \mathrm{d}$. 


\section{Interrelationships of coalition stabilities with pref- erence uncertainty}

For the four solution concepts, Nash, GMR, SMR, and SEQ, different interrelationships exist for individual stabilities with both simple preference [3] and uncertain preference [14]. This section examines interrelationships of coalition stabilities with preference uncertainty and the examination starts with interrelationships among the four coalition stabilities within each extension.

Theorem 1 Interrelationships of coalition stability for $H$ within each extension. For $a$ particular extension $k \in\{a, b, c, d\}$ and a nonempty $H \subseteq N, S_{H}^{C N a s h_{k}} \subseteq S_{H}^{C S E Q_{k}} \subseteq S_{H}^{C G M R_{k}}$ and $S_{H}^{C N a s h_{k}} \subseteq S_{H}^{C S M R_{k}} \subseteq S_{H}^{C G M R_{k}}$.

Proof: The proof will be carried out for extension a only and the remaining three extensions can be proved similarly.

Firstly, for a state $s \in S$ and a nonempty $H \subseteq N$, if $s \in S_{H}^{C N a s h_{a}}$, Definition 9 implies that $C R_{H}^{+, U}(s)=\varnothing$. Then it automatically follows that $s \in S_{H}^{C G M R_{a}}, s \in S_{H}^{C S M R_{a}}$ and $s \in S_{H}^{C S E Q_{a}}$. Hence, $S_{H}^{C N a s h_{a}} \subseteq S_{H}^{C G M R_{a}}, S_{H}^{C N a s h_{a}} \subseteq S_{H}^{C S M R_{a}}$, and $S_{H}^{C N a s h_{a}} \subseteq S_{H}^{C S E Q_{a}}$.

Next, we prove that $S_{H}^{C S E Q_{a}} \subseteq S_{H}^{C G M R_{a}}$. For any state $s \in S_{H}^{C S E Q_{a}}$, either $C R_{H}^{+, U}(s)=\varnothing$ or $C R_{H}^{+, U}(s) \neq \varnothing$. If $C R_{H}^{+, U}(s)=\varnothing$, then $s \in S_{H}^{C N a s h_{a}} \subseteq S_{H}^{C G M R_{a}}$ based on the aforesaid argument. Next, we examine the case that $C R_{H}^{+, U}(s) \neq \varnothing$. As per Definition 13, for every $s_{1} \in C R_{H}^{+, U}(s)$, there exists $s_{2} \in R_{N-H}^{+, U}\left(s_{1}\right)$ such that $s_{2} \in \Phi_{H}^{\leq}(s)$. By Definition 2, $R_{N-H}^{+, U}\left(s_{1}\right) \subseteq R_{N-H}\left(s_{1}\right)$, implying that $s \in S_{H}^{C G M R_{a}}$.

Lastly, we certify that $S_{H}^{C S M R_{a}} \subseteq S_{H}^{C G M R_{a}}$. For any state $s \in S_{H}^{C S M R_{a}}$, either $C R_{H}^{+, U}(s)=$ $\varnothing$ or $C R_{H}^{+, U}(s) \neq \varnothing$. If $C R_{H}^{+, U}(s)=\varnothing$, then $s \in S_{H}^{C N a s h_{a}} \subseteq S_{H}^{C G M R_{a}}$ as certified above. If $C R_{H}^{+, U}(s) \neq \varnothing$, based on Definition 12, for every $s_{1} \in C R_{H}^{+, U}(s)$, there exists $s_{2} \in R_{N-H}\left(s_{1}\right)$ such that $s_{2} \in \Phi_{H}^{\leq}(s)$ and $s_{3} \in \Phi_{H}^{\leq}(s)$ for all $s_{3} \in R_{H}\left(s_{2}\right)$. Without considering $H$ 's response $s_{3}$, one obtains $s \in S_{H}^{C G M R_{a}}$.

It should be noted that $S_{H}^{C G M R_{a}} \nsubseteq S_{H}^{C S M R_{a}}$. The reason is as follows: If $s \in S_{H}^{C G M R_{a}}$, for every $s_{1} \in C R_{H}^{+, U}(s)$, there exists $s_{2} \in R_{N-H}\left(s_{1}\right)$ such that $s_{2} \in \Phi_{H}^{\leq}(s)$. However, 
as long as for a particular $s_{2}$, there exists $s_{3} \in R_{H}\left(s_{2}\right)$ such that $s_{3} \in C R_{H}^{+, U}(s)$, then $s \notin S_{H}^{C S M R_{a}}$.

Similarly, $S_{H}^{C G M R_{a}} \nsubseteq S_{H}^{C S E Q_{a}}$. Once again, if $s \in S_{H}^{C G M R_{a}}$, for every $s_{1} \in C R_{H}^{+, U}(s)$, there exists $s_{2} \in R_{N-H}\left(s_{1}\right)$ such that $s_{2} \in \Phi_{H}^{\leq}(s)$. In this case, if for a particular $s_{1} \epsilon$ $C R_{H}^{+, U}(s), R_{N-H}^{+, U}\left(s_{1}\right)=\varnothing$, then $s \notin S_{H}^{C S E Q_{a}}$.

In addition, there generally does not exist any inclusion relationship between $S_{H}^{C S E Q_{a}}$ and $S_{H}^{C S M R_{a}}$, either.

Theorem 1 ascertains the interrelationships for the four coalition stabilities for a particular nonempty coalition within each extension. Given that for any extension $k$, $S^{C G M R_{k}}=\cap_{H \subseteq N} S_{H}^{C G M R_{k}}, S^{C S M R_{k}}=\cap_{H \subseteq N} S_{H}^{C S M R_{k}}$, and $S^{C S E Q_{k}}=\cap_{H \subseteq N} S_{H}^{C S E Q_{k}}, k=\mathrm{a}, \mathrm{b}$, c, d, the following corollary immediately follows:

Corollary 1 Interrelationships of coalition stability within each extension For a particular extension $k \in\{a, b, c, d\}, S^{C N a s h_{k}} \subseteq S^{C S E Q_{k}} \subseteq S^{C G M R_{k}}$ and $S^{C N a s h_{k}} \subseteq S^{C S M R_{k}} \subseteq$ $S^{C G M R_{k}}$.

The interrelationships of the four coalition stabilities within each extension can thus be depicted in a Venn diagram as shown in Fig. 1. The result here is consistent with the interrelationships for individual stabilities with simple preference [3] and uncertain preference [14] as well as coalition stabilities with simple preference [9].

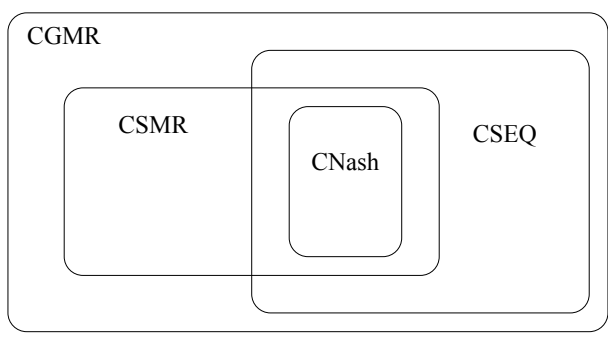

Figure 1: Interrelationships of coalition stabilities within each extension

Next, the interrelationships of each coalition stability across the four extensions will be investigated. First, for coalition Nash stability, the following result holds true.

Theorem 2 Interrelationships of coalition Nash stability for $H$ across extensions. For a nonempty $H \subseteq N, S_{H}^{C N a h_{a}}=S_{H}^{C N a s h_{c}}, S_{H}^{C N a s h_{b}}=S_{H}^{C N a s h_{d}}$, and $S_{H}^{C N a s h_{a}} \subseteq S_{H}^{C N a s h_{b}}$. 
Proof. When Definitions 9 and 10 are introduced, it has been mentioned that $S_{H}^{C N a s h_{a}}=S_{H}^{C N a s h_{c}}, S_{H}^{C N a s h_{b}}=S_{H}^{C N a s h_{d}}$. Now, we shall confirm that $S_{H}^{C N a s h_{a}} \subseteq S_{H}^{C N a s h_{b}}$.

For $H \subseteq N$ and any $s \in S$, if $s \in S_{H}^{C N a s h_{a}}$, by Definition $9, R_{H}^{+, U}(s)=\varnothing$. As $R_{H}^{+}(s) \subseteq$ $R_{H}^{+, U}(s)$, it follows that $R_{H}^{+}(s)=\varnothing$, implying that $s \in S_{H}^{C N a s h_{b}}$.

Similarly, Corollary 2 immediately follows.

Corollary 2 Interrelationships of coalition Nash stability across extensions. $S^{C N a s h_{a}}=$ $S^{C N a s h_{c}}, S^{C N a s h_{b}}=S^{C N a s h_{d}}$, and $S^{C N a s h_{a}} \subseteq S^{C N a s h_{b}}$.

The interrelationships of coalition Nash stabilities across the four extensions can be illustrated as a Venn diagram in Fig. 2.

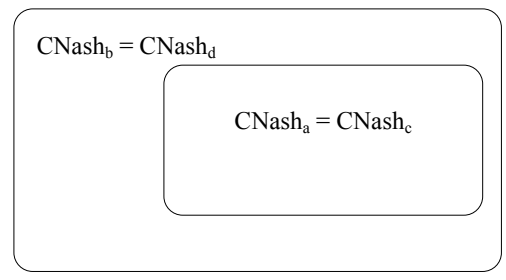

Figure 2: Interrelationships of coalition Nash stabilities across extensions

Next, interrelationships across the four extensions will be investigated for the remaining three coalition stabilities (CGMR, CSMR, and CSEQ) with preference uncertainty. For conciseness, let CSC be a generic coalition solution concept, which can be CGMR, CSMR, or CSEQ.

Theorem 3 Interrelationships of CGMR, CSMR, and CSEQ stabilities for $H$ across four extensions. For a nonempty $H \subseteq N$ and a particular $C S C, S_{H}^{C S C_{a}} \subseteq S_{H}^{C S C_{b}} \subseteq S_{H}^{C S C_{d}}$ and $S_{H}^{C S C_{a}} \subseteq S_{H}^{C S C_{c}} \subseteq S_{H}^{C S C_{d}}$, where CSC $=$ CGMR, CSMR, CSEQ.

Proof. The proof will only be carried out with coalition GMR stability. The interrelationships for the other two coalition stabilities can be similarly proved.

First, we shall prove that, for an $H \subseteq N$ and any $s \in S$, if $s \in S_{H}^{C G M R_{a}}$, then $s \in$ $S_{H}^{C G M R_{b}}, s \in S_{H}^{C G M R_{c}}$, and $s \in S_{H}^{C G M R_{d}}$.

If $s \in S_{H}^{C G M R_{a}}$ and $C R_{H}^{+, U}(s)=\varnothing$, then $s \in S_{H}^{C N a s h_{a}}=S_{H}^{C N a s h_{c}} \subseteq S_{H}^{C N a s h_{b}}=S_{H}^{C N a s h_{d}}$ as per Definition 9 and Theorem 2. Based on Theorem 1, it is confirmed that $s \in S_{H}^{C G M R_{b}}, s \in$ $S_{H}^{C G M R_{c}}$, and $s \in S_{H}^{C G M R_{d}}$. 
Next, we consider the case that $C R_{H}^{+, U} \neq \varnothing$. Two cases may arise: if $C R_{H}^{+}(s)=\varnothing$, by Definition 10, $s \in S_{H}^{N a s h_{b}}$ and, hence, $s \in S_{H}^{C G M R_{b}}$ by Theorem 1. Otherwise, if $C R_{H}^{+}(s) \neq$ $\varnothing$, as $s \in S_{H}^{C G M R_{a}}$, for every $s_{1} \in C R_{H}^{+}(s) \subseteq C R_{H}^{+, U}(s)$, there exists $s_{2} \in R_{N-H}\left(s_{1}\right)$ such that $s_{2} \in \Phi_{H}^{\leq}(s)$. By Definition 14, $s \in S_{H}^{C G M R_{b}}$.

For extension c, since $s \in S_{H}^{C G M R_{a}}$, for any $s_{1} \in C R_{H}^{+}(s) \subseteq C R_{H}^{+, U}(s)$, there exists $s_{2} \in R_{N-H}\left(s_{1}\right)$ such that $s_{2} \in \Phi_{H}^{\leq}(s) \subseteq \Phi_{H}^{\leq, U}(s)$, implying that $s \in S_{H}^{C G M R_{c}}$.

For extension d, the case of $C R_{H}^{+}(s)=\varnothing$ is trivial and we only consider $C R_{H}^{+}(s) \neq \varnothing$. For any $s_{1} \in C R_{H}^{+}(s) \subseteq C R_{H}^{+, U}(s)$, as $s \in S_{H}^{C G M R_{a}}$, there exists $s_{2} \in R_{N-H}\left(s_{1}\right)$ such that $s_{2} \in \Phi_{H}^{\leq}(s)$. By Definition 20, $s \in S_{H}^{C G M R_{d}}$.

Next, we shall prove that, for any $s \in S$, if $s \in S_{H}^{C G M R_{b}}$, then $s \in S_{H}^{C G M R_{d}}$. If $s \in S_{H}^{C G M R_{b}}$ and $C R_{H}^{+}(s)=\varnothing$, then $s \in S_{H}^{C N a h_{b}}=S_{H}^{C N a s h_{d}}$ as per Definition 10 and Theorem 2. By Theorem 1, we have $s \in S_{H}^{C G M R_{d}}$.

The following argument assumes that $C R_{H}^{+}(s) \neq \varnothing$. Since $s \in S_{H}^{C G M R_{b}}$, for any $s_{1} \epsilon$ $C R_{H}^{+}(s)$, there exists $s_{2} \in R_{N-H}\left(s_{1}\right)$ such that $s_{2} \in \Phi_{H}^{\leq}(s)$. As $\Phi_{H}^{\leq}(s) \subseteq \Phi_{H}^{\leq, U}(s)$, by Definition 20, $s \in S_{H}^{C G M R_{d}}$.

Lastly, we shall verify that $S_{H}^{C G M R_{c}} \subseteq S_{H}^{C G M R_{d}}$. For any $s \in S_{H}^{C G M R_{c}}$, either $C R_{H}^{+}(s)=$ $\varnothing$ or $C R_{H}^{+}(s) \neq \varnothing$. If $C R_{H}^{+}(s)=\varnothing$, then $s \in S_{H}^{C N a s h_{b}}=S_{H}^{C N a s h_{d}}$ by Definition 10 and Theorem 2. If $C R_{H}^{+}(s) \neq \varnothing$, for every $s_{1} \in C R_{H}^{+}(s)$, we also have $s_{1} \in C R_{H}^{+, U}(s)$ due to $C R_{H}^{+}(s) \subseteq C R_{H}^{+, U}(s)$. Since $s \in S_{H}^{C G M R_{c}}$, there exists $s_{2} \in R_{N-H}\left(s_{1}\right)$ such that $s_{2} \in \Phi_{H}^{\leq, U}(s)$. This completes the proof of $S_{H}^{C G M R_{c}} \subseteq S_{H}^{C G M R_{d}}$.

The aforesaid argument proves that $S_{H}^{C G M R_{a}} \subseteq S_{H}^{C G M R_{b}} \subseteq S_{H}^{C G M R_{d}}$ and $S_{H}^{C G M R_{a}} \subseteq$ $S_{H}^{C G M R_{c}} \subseteq S_{H}^{C G M R_{d}}$.

It is apparent that the following corollary holds true.

Corollary 3 Interrelationships of CGMR, CSMR, and CSEQ stabilities across four extensions. For a particular $C S C, S^{C S C_{a}} \subseteq S^{C S C_{b}} \subseteq S^{C S C_{d}}$ and $S^{C S C_{a}} \subseteq S^{C S C_{c}} \subseteq S^{C S C_{d}}$, where $C S C=C G M R, C S M R, C S E Q$.

The interrelationships of CGMR, CSMR, CSEQ stabilities revealed in Theorem 3 and Corollary 3 can thus be illustrated in Fig. 3 . 


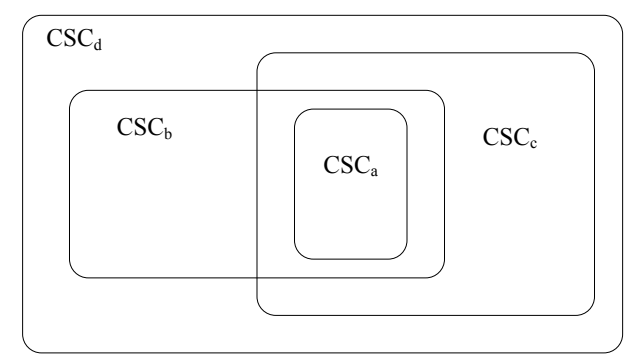

Figure 3: Interrelationships of coalition stabilities across extensions, where CSC = CGMR, CSMR, CSEQ

\section{A case study: Coalition analysis for the Gisborne conflict}

The conflict surrounding the Lake Gisborne bulk-water export issues was first established as a three-DM graph model by Fang et al. [4]. The conflict arose in June 1995 when a local company proposed to export bulk water from Lake Gisborne located in the Province of Newfoundland and Labrador in Canada. The project was approved by the provincial government in anticipation of its economic benefit in December 1996, but this initial approval was heavily criticized by a number of lobby groups, citing unpredictable detriments to the local environment and culture. This opposition was subsequently echoed by the Canadian federal government with the introduction of a new policy to prevent water export from major drainage basins in Canada. The mounting pressure led the provincial government to withdraw its support and introduce a ban on bulk water export from Newfoundland and Labrador in late 1999. This development seemingly brought the conflict to a conclusion. However, in March 2001 when a new provincial government took the office, the newly elected premier initiated a review of the Gisborne project. This move received immediate attention from the opposition group. Although the tension was soon eased by a reiteration of the ban in a government report released on October 18, 2001, the evolution of events surrounding the Gisborne project indicates that this conflict may arise again in the future if potential revenue from water export creates a strong enough incentive for an economically-oriented provincial government to deviate from its current position. For more details about the development of this conflict, readers are referred to [4] and [14]. 
Table 1: DMs and options in the Gisborne conflict model [14]

\begin{tabular}{l|l}
\hline \multicolumn{1}{c|}{ DMs } & \multicolumn{1}{c}{ Options } \\
\hline DM 1: Federal government (Federal) & $\begin{array}{l}\text { 1. Continue a Canada wide ac- } \\
\text { cord on the prohibition of bulk } \\
\text { water export (Continue) }\end{array}$ \\
\hline $\begin{array}{l}\text { DM 2: Provincial government of New- } \\
\text { foundland and Labrador (Provincial) }\end{array}$ & $\begin{array}{l}\text { 2. Lift the ban on bulk water ex- } \\
\text { port (Lift) }\end{array}$ \\
\hline DM 3: Support groups (Support) & $\begin{array}{l}\text { 3. Appeal for continuing the Gis- } \\
\text { borne project (Appeal) }\end{array}$ \\
\hline
\end{tabular}

A graph model was developed for this conflict with three DMs and each DM controlling one option as shown in Table 1. In this model, DM 1 includes both the Federal Government of Canada and opposition groups, DM 2 is self-evident, and DM 3 stands for both the firm that proposed the Gisborne project and other groups that support bulk water export from Canada. A brief explanation is furnished in Table 1 for each option controlled by the corresponding DM.

The resulting eight feasible states are given in Table 2, where a "Y" opposite an option indicates that the option is selected by the controlling DM and an " $\mathrm{N}$ " indicates the corresponding option is not chosen by the DM.

Table 2: Feasible states for the Gisborne conflict model [14]

\section{Federal}

\begin{tabular}{|c|c|c|c|c|c|c|c|c|}
\hline 1. Continue & $\mathrm{N}$ & $\mathrm{Y}$ & $\mathrm{N}$ & $\mathrm{Y}$ & $\mathrm{N}$ & $Y$ & $\mathrm{~N}$ & $\mathrm{Y}$ \\
\hline Provincial & \multirow{3}{*}{$\mathrm{N}$} & \multirow{3}{*}{$\mathrm{N}$} & \multirow{3}{*}{$\mathrm{Y}$} & \multirow{3}{*}{ Y } & \multirow{3}{*}{$\mathrm{N}$} & & \multirow{3}{*}{$\mathrm{Y}$} & \\
\hline 2. Lift & & & & & & \multirow[t]{2}{*}{$\mathrm{N}$} & & Y \\
\hline Support & & & & & & & & \\
\hline 3. Appeal & $\mathrm{N}$ & $\mathrm{N}$ & $\mathrm{N}$ & $\mathrm{N}$ & $\mathrm{Y}$ & $Y$ & $\mathrm{Y}$ & $\mathrm{Y}$ \\
\hline States & $s_{1}$ & $s_{2}$ & $s_{3}$ & $s_{4}$ & $s_{5}$ & $s_{t}$ & $s_{7}$ & $s_{8}$ \\
\hline
\end{tabular}

To model the oscillation of the provincial government's attitude towards the Gisborne bulk water export conflict, Li et al. [14] introduced uncertain preference for DM 2 Provincial. The relative preference information for this conflict model is furnished in Table 3. For DM 2, except for the strict preference indicated in the four pairs of states enclosed 
within the curly brackets, remaining preference relations are assumed to be uncertain between any two states from any two different curly brackets. For instance, the provincial government prefers state $s_{3}$ to $s_{7}$, but is uncertain about its preference between $s_{3}$ and any other states.

Table 3: Preference information for the Gisborne model [14]

\begin{tabular}{l|l}
\hline \multicolumn{1}{c|}{ DMs } & \multicolumn{1}{|c}{ Relative preference } \\
\hline 1. Federal & $s_{2}>s_{6}>s_{4}>s_{8}>s_{1}>s_{5}>s_{3}>s_{7}$ \\
\hline 2. Provincial & $\left\{s_{3}>s_{7}\right\} U\left\{s_{4}>s_{8}\right\} U\left\{s_{1}>s_{5}\right\} U\left\{s_{2}>s_{6}\right\}$ \\
\hline 3. Support & $s_{3}>s_{4}>s_{7}>s_{8}>s_{5}>s_{6}>s_{1}>s_{2}$ \\
\hline
\end{tabular}

An integrated graph of this conflict model is illustrated in Fig. 4 where the nodes correspond to the eight states and the DMs are labeled on the arcs to indicate the moves controlled by the DMs.

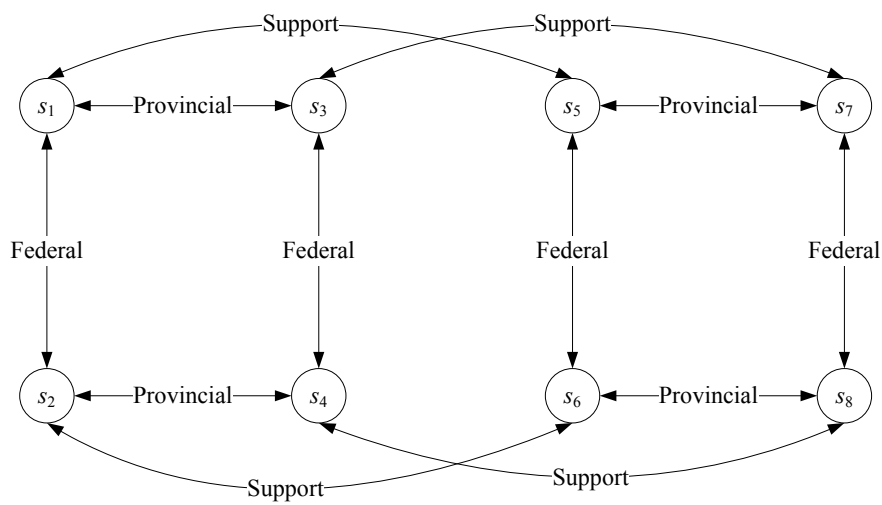

Figure 4: An integrated graph of the Gisborne conflict model [14]

Based on the moves controlled by each DM given in Fig. 4, one can obtain the reachable list for each of the seven nonempty coalitions as shown in Table 4, where a particular coalition is identified by an appropriate subscript in columns 2 through 8 . This coalition reachable list information is needed for determining coalition improvement lists and coalition improvement or uncertain move lists as well as examining countermoves by an opponent coalition in assessing CGMR and CSMR stabilities.

By incorporating DMs' preference information into the coalition reachable lists, the following coalition improvement or uncertain move lists can be derived as given in Table 
Table 4: Coalition reachable lists for the Gisborne model

\begin{tabular}{c|c|c|c|c|c|c|c}
\hline$s$ & $R_{\{1\}}(s)$ & $R_{\{2\}}(s)$ & $R_{\{3\}}(s)$ & $R_{\{1,2\}}(s)$ & $R_{\{1,3\}}(s)$ & $R_{\{2,3\}}(s)$ & $R_{\{1,2,3\}}(s)$ \\
\hline$s_{1}$ & $s_{2}$ & $s_{3}$ & $s_{5}$ & $s_{2}, s_{3}, s_{4}$ & $s_{2}, s_{5}, s_{6}$ & $s_{3}, s_{5}, s_{7}$ & $s_{2}, s_{3}, s_{4}, s_{5}, s_{6}, s_{7}, s_{8}$ \\
\hline$s_{2}$ & $s_{1}$ & $s_{4}$ & $s_{6}$ & $s_{1}, s_{3}, s_{4}$ & $s_{1}, s_{5}, s_{6}$ & $s_{4}, s_{6}, s_{8}$ & $s_{1}, s_{3}, s_{4}, s_{5}, s_{6}, s_{7}, s_{8}$ \\
\hline$s_{3}$ & $s_{4}$ & $s_{1}$ & $s_{7}$ & $s_{1}, s_{2}, s_{4}$ & $s_{4}, s_{7}, s_{8}$ & $s_{1}, s_{5}, s_{7}$ & $s_{1}, s_{2}, s_{4}, s_{5}, s_{6}, s_{7}, s_{8}$ \\
\hline$s_{4}$ & $s_{3}$ & $s_{2}$ & $s_{8}$ & $s_{1}, s_{2}, s_{3}$ & $s_{3}, s_{7}, s_{8}$ & $s_{2}, s_{6}, s_{8}$ & $s_{1}, s_{2}, s_{3}, s_{5}, s_{6}, s_{7}, s_{8}$ \\
\hline$s_{5}$ & $s_{6}$ & $s_{7}$ & $s_{1}$ & $s_{6}, s_{7}, s_{8}$ & $s_{1}, s_{2}, s_{6}$ & $s_{1}, s_{3}, s_{7}$ & $s_{1}, s_{2}, s_{3}, s_{4}, s_{6}, s_{7}, s_{8}$ \\
\hline$s_{6}$ & $s_{5}$ & $s_{8}$ & $s_{2}$ & $s_{5}, s_{7}, s_{8}$ & $s_{1}, s_{2}, s_{5}$ & $s_{2}, s_{4}, s_{8}$ & $s_{1}, s_{2}, s_{3}, s_{4}, s_{5}, s_{7}, s_{8}$ \\
\hline$s_{7}$ & $s_{8}$ & $s_{5}$ & $s_{3}$ & $s_{5}, s_{6}, s_{8}$ & $s_{3}, s_{4}, s_{8}$ & $s_{1}, s_{3}, s_{5}$ & $s_{1}, s_{2}, s_{3}, s_{4}, s_{5}, s_{6}, s_{8}$ \\
\hline$s_{8}$ & $s_{7}$ & $s_{6}$ & $s_{4}$ & $s_{5}, s_{6}, s_{7}$ & $s_{3}, s_{4}, s_{7}$ & $s_{2}, s_{4}, s_{6}$ & $s_{1}, s_{2}, s_{3}, s_{4}, s_{5}, s_{6}, s_{7}$ \\
\hline
\end{tabular}

Table 5: Coalition improvement or uncertain move lists for the Gisborne model

\begin{tabular}{c|c|c|c|c|c|c|c}
\hline$s$ & $C R_{\{1\}}^{+, U}(s)$ & $C R_{\{2\}}^{+, U}(s)$ & $C R_{\{3\}}^{+, U}(s)$ & $C R_{\{1,2\}}^{+, U}(s)$ & $C R_{\{1,3\}}^{+, U}(s)$ & $C R_{\{2,3\}}^{+, U}(s)$ & $C R_{\{1,2,3\}}^{+, U}(s)$ \\
\hline$s_{1}$ & $\bar{s}_{2}$ & $s_{3}$ & $\bar{s}_{5}$ & $s_{2}, s_{4}$ & $\bar{s}_{6}$ & $s_{3}, s_{7}$ & $s_{4}, s_{6}, s_{8}$ \\
\hline$s_{2}$ & - & $s_{4}$ & $\bar{s}_{6}$ & - & - & $s_{4}, s_{8}$ & - \\
\hline$s_{3}$ & $\bar{s}_{4}$ & $s_{1}$ & - & $s_{1}, s_{2}, s_{4}$ & - & - & - \\
\hline$s_{4}$ & - & $s_{2}$ & - & $s_{2}$ & - & - & - \\
\hline$s_{5}$ & $\bar{s}_{6}$ & $s_{7}$ & - & $s_{6}, s_{8}$ & - & $s_{3}, s_{7}$ & $s_{4}, s_{8}$ \\
\hline$s_{6}$ & - & $s_{8}$ & - & - & - & $s_{4}, s_{8}$ & - \\
\hline$s_{7}$ & $\bar{s}_{8}$ & $s_{5}$ & $\bar{s}_{3}$ & $s_{5}, s_{6}, s_{8}$ & $\bar{s}_{3}, \bar{s}_{4}$ & $\bar{s}_{3}$ & $\bar{s}_{3}, s_{4}$ \\
\hline$s_{8}$ & - & $s_{6}$ & $\bar{s}_{4}$ & $s_{6}$ & $\bar{s}_{4}$ & $\bar{s}_{4}$ & $\bar{s}_{4}$ \\
\hline
\end{tabular}

5, where a "-" indicates an empty CIUM list and a coalition improvement is highlighted with an overline for the corresponding state in a CIUM list. For instance, from Table 5, one can have $R_{\{1,2,3\}}^{+, U}\left(s_{7}\right)=\left\{s_{3}, s_{4}\right\}$ but $R_{\{1,2,3\}}^{+}\left(s_{7}\right)=\left\{s_{3}\right\}$ as only $s_{3}$ is highlighted by an overline, indicating that it is a coalition improvement for the grand coalition $N=\{1,2,3\}$ relative to the status quo state $s_{7}$. Similarly, Table 5 shows that $R_{\{1,2\}}^{+, U}\left(s_{1}\right)=\left\{s_{2}, s_{4}\right\}$ but $R_{\{1,2\}}^{+}\left(s_{1}\right)=\varnothing$ as none of the CIUMs starting from $s_{1}$ is identified as a coalition improvement for $H=\{1,2\}$ by an overline. The information in Table 5 will play a significant role in determining whether a focal coalition will be possibly motivated to deviate from a status quo in coalition stability.

In assessing CSEQ stability, one has to examine the credibility of the sanction by the 
opponents $N-H$. Table 6 furnishes this information. Since a nonempty coalition will not be examined for CSEQ stability, its opponents, the grand coalition $N$, is thus excluded from Table 6.

Table 6: Unilateral improvement or uncertain move lists by a coalition for the Gisborne model

\begin{tabular}{c|c|c|c|c|c|c}
\hline$s$ & $R_{\{1\}}^{+, U}(s)$ & $R_{\{2\}}^{+, U}(s)$ & $R_{\{3\}}^{+, U}(s)$ & $R_{\{1,2\}}^{+, U}(s)$ & $R_{\{1,3\}}^{+, U}(s)$ & $R_{\{2,3\}}^{+, U}(s)$ \\
\hline$s_{1}$ & $s_{2}$ & $s_{3}$ & $s_{5}$ & $s_{2}, s_{3}, s_{4}$ & $s_{2}, s_{5}, s_{6}$ & $s_{3}, s_{5}, s_{7}$ \\
\hline$s_{2}$ & - & $s_{4}$ & $s_{6}$ & $s_{4}$ & $s_{6}$ & $s_{4}, s_{6}, s_{8}$ \\
\hline$s_{3}$ & $s_{4}$ & $s_{1}$ & - & $s_{1}, s_{2}, s_{4}$ & $s_{4}$ & $s_{1}, s_{5}, s_{7}$ \\
\hline$s_{4}$ & - & $s_{2}$ & - & $s_{2}$ & - & $s_{2}, s_{6}, s_{8}$ \\
\hline$s_{5}$ & $s_{6}$ & $s_{7}$ & - & $s_{6}, s_{7}, s_{8}$ & $s_{6}$ & $s_{1}, s_{3}, s_{7}$ \\
\hline$s_{6}$ & - & $s_{8}$ & - & $s_{8}$ & - & $s_{2}, s_{4}, s_{8}$ \\
\hline$s_{7}$ & $s_{8}$ & $s_{5}$ & $s_{3}$ & $s_{5}, s_{6}, s_{8}$ & $s_{3}, s_{4}, s_{8}$ & $s_{1}, s_{3}, s_{5}$ \\
\hline$s_{8}$ & - & $s_{6}$ & $s_{4}$ & $s_{6}$ & $s_{4}$ & $s_{2}, s_{4}, s_{6}$ \\
\hline
\end{tabular}

By utilizing the information in Tables 4, 5, and 6, one can conduct a coalition analysis for CNash, CGMR, CSMR, and CSEQ under the four extensions as defined in Sections 2 and 3. As mentioned earlier, this paper treats coalition analysis as a follow-up analysis after a standard stability analysis. Therefore, only predicted equilibria under individual calculations are examined for coalition stability.

A stability analysis by $\mathrm{Li}$ et al. [14] reveals that states $s_{4}, s_{6}$, and $s_{8}$ possess some equilibrium status under various circumstances. As this research treats coalition analysis as a follow-up analysis after a standard stability analysis, coalition stability is examined for these three states only. The analysis result is summarized in Tables 7 (for CNash and CGMR stabilities) and 8 (for CSMR and CSEQ stabilities). For the sake of space, each coalition is simply identified by the corresponding $\mathrm{DM}(\mathrm{s})$ without curly brackets. For instance, 12 in the second row of Tables 7 and 8 stands for coalition $H=\{1,2\}$. A $\sqrt{ }$ in the column of a particular coalition in Tables 7 and 8 indicates that a state is stable for this coalition under a specific extension of a coalition solution concept (CSC). If a state is stable for all nonempty coalitions under an extension of a CSC, a $\sqrt{ }$ is placed in the appropriate cell in the column for the particular CSC, indicating that the 
state is coalitionally stable for this CSC in terms of the specific extension. For instance, Table 7 illustrates that states $s_{4}$ and $s_{6}$ are coalitionally conservatively Nash stable (under extensions b and d) and coalitionally GMR stable under extensions b, c, and d. Similarly, Table 8 shows that states $s_{4}$ and $s_{6}$ are coalitionally SMR stable under extensions b, c, and d, but coalitionally SEQ stable for extensions b and d only.

Tables 7 and 8 confirm the interrelationships of the four coalition solution concepts, CNash, CGMR, CSMR, and CSEQ, within each extension and across the four extensions as described in the previous section.

Coalition analysis results in Tables 7 and 8 also shed structural insights into the Gisborne conflict. More specifically, states $s_{4}$ and $s_{6}$ are Nash, GMR, SMR, and SEQ equilibria under extensions b and $\mathrm{d}$ as ascertained by Li et al. [14], our analysis here further confirms that they remain coalitionally stable for the four solution concepts under these two extensions. A common feature between extensions $\mathrm{b}$ and $\mathrm{d}$ is that the first mover (either an individual DM or a coalition) is conservative towards preference uncertainty and is unwilling to depart for uncertain outcomes. Therefore, as long as an analyst is confident that the DMs in this conflict are conservative at the incentive end, states $s_{4}$ and $s_{6}$ will be sustainable resolutions under both individual and coalition considerations.

[14] also indicates that states $s_{4}$ and $s_{6}$ are GMR, SMR, and SEQ equilibria under extension c. Table 7 and 8 show that they remain coalitionally GMR and SMR stable under this extension, but only $s_{4}$ is coalitionally SEQ stable. Equilibrium $s_{6}$ can be upset by DMs 2 and 3 forming a coalition and coordinating their moves (Provincial government sides with the support group and DMs are aggressive at the incentive end). In this case, their CIUMs from state $s_{6}$ are $s_{4}$ and $s_{8}$, which leave their opponent Federal without any credible sanction. Given that coalition analysis is a post-stability analysis and state $s_{8}$ is not an SEQ equilibrium, a viable coalition move is an equilibrium jump from $s_{6}$ to $s_{4}$, i.e., $s_{6} \longrightarrow_{2} s_{8} \longrightarrow_{3} s_{4}$ or $s_{6} \longrightarrow_{3} s_{2} \longrightarrow_{2} s_{4}$, where a subscript of an arrow indicates a DM controlling a particular move. If the first mover (an individual DM or a coalition) believes that its opponents are also concerned with their welfare in their response as depicted in the SEQ stability, the only sustainable resolution of this conflict 
would be state $s_{4}$ provided that the DMs' risk profile towards preference uncertainty can be characterized by extension c.

[14] further reveals that state $s_{8}$ is GMR and SMR equilibrium under extensions b, c, and d. Our analysis here indicates that this equilibrium will not survive coalition moves for CGMR or CSMR under any of the three extensions. The implication is that equilibrium $s_{8}$ is likely a transient resolution and may be overturned by coalition moves. Since $s_{4}$ is preferred by all DMs relative to $s_{8}$ and has the strongest individual and coalition stability status in this model, an equilibrium jump from $s_{8}$ to $s_{4}$ seems to be the most likely outcome.

Table 7: Coalition analysis result for CNash and CGMR for the Gisborne model

\begin{tabular}{|c|c|c|c|c|c|c|c|c|c|c|c|c|c|c|c|c|c|}
\hline \multirow{2}{*}{\multicolumn{2}{|c|}{ State }} & \multicolumn{7}{|c|}{$\mathrm{CNash}_{H}$} & \multirow{2}{*}{ CNash } & \multicolumn{7}{|c|}{$\mathrm{CGMR}_{H}$} & \multirow{2}{*}{ CGMR } \\
\hline & & 1 & 2 & 3 & 12 & 13 & 23 & 123 & & 1 & 2 & 3 & 12 & 13 & 23 & 123 & \\
\hline & $\mathrm{a}$ & $\sqrt{ }$ & & $\sqrt{ }$ & & $\sqrt{ }$ & $\sqrt{ }$ & $\sqrt{ }$ & & $\sqrt{ }$ & & $\sqrt{ }$ & & $\sqrt{ }$ & $\sqrt{ }$ & $\sqrt{ }$ & \\
\hline \multirow{3}{*}{$S_{4}$} & $\mathrm{~b}$ & $\sqrt{ }$ & $\sqrt{ }$ & $\sqrt{ }$ & $\sqrt{ }$ & $\sqrt{ }$ & $\sqrt{ }$ & $\sqrt{ }$ & $\sqrt{ }$ & $\sqrt{ }$ & $\sqrt{ }$ & $\sqrt{ }$ & $\sqrt{ }$ & $\sqrt{ }$ & $\sqrt{ }$ & $\sqrt{ }$ & $\sqrt{ }$ \\
\hline & c & $\sqrt{ }$ & & $\sqrt{ }$ & & $\sqrt{ }$ & $\sqrt{ }$ & $\sqrt{ }$ & & $\sqrt{ }$ & $\sqrt{ }$ & $\sqrt{ }$ & $\sqrt{ }$ & $\sqrt{ }$ & $\sqrt{ }$ & $\sqrt{ }$ & $\sqrt{ }$ \\
\hline & d & $\sqrt{ }$ & $\sqrt{ }$ & $\sqrt{ }$ & $\sqrt{ }$ & $\sqrt{ }$ & $\sqrt{ }$ & $\sqrt{ }$ & $\sqrt{ }$ & $\sqrt{ }$ & $\sqrt{ }$ & $\sqrt{ }$ & $\sqrt{ }$ & $\sqrt{ }$ & $\sqrt{ }$ & $\sqrt{ }$ & $\sqrt{ }$ \\
\hline & $\mathrm{a}$ & $\sqrt{ }$ & & $\sqrt{ }$ & $\sqrt{ }$ & $\sqrt{ }$ & & $\sqrt{ }$ & & $\sqrt{ }$ & & $\sqrt{ }$ & $\sqrt{ }$ & $\sqrt{ }$ & & $\sqrt{ }$ & \\
\hline \multirow{3}{*}{$s_{6}$} & $\mathrm{~b}$ & $\sqrt{ }$ & $\sqrt{ }$ & $\sqrt{ }$ & $\sqrt{ }$ & $\sqrt{ }$ & $\sqrt{ }$ & $\sqrt{ }$ & $\sqrt{ }$ & $\sqrt{ }$ & $\sqrt{ }$ & $\sqrt{ }$ & $\sqrt{ }$ & $\sqrt{ }$ & $\sqrt{ }$ & $\sqrt{ }$ & $\sqrt{ }$ \\
\hline & c & $\sqrt{ }$ & & $\sqrt{ }$ & $\sqrt{ }$ & $\sqrt{ }$ & & $\sqrt{ }$ & & $\sqrt{ }$ & $\sqrt{ }$ & $\sqrt{ }$ & $\sqrt{ }$ & $\sqrt{ }$ & $\sqrt{ }$ & $\sqrt{ }$ & $\sqrt{ }$ \\
\hline & d & $\sqrt{ }$ & $\sqrt{ }$ & $\sqrt{ }$ & $\sqrt{ }$ & $\sqrt{ }$ & $\sqrt{ }$ & $\sqrt{ }$ & $\sqrt{ }$ & $\sqrt{ }$ & $\sqrt{ }$ & $\sqrt{ }$ & $\sqrt{ }$ & $\sqrt{ }$ & $\sqrt{ }$ & $\sqrt{ }$ & $\sqrt{ }$ \\
\hline & $\mathrm{a}$ & $\sqrt{ }$ & & & & & & & & $\sqrt{ }$ & & $\sqrt{ }$ & & $\sqrt{ }$ & & & \\
\hline & $\mathrm{b}$ & $\sqrt{ }$ & $\sqrt{ }$ & & $\sqrt{ }$ & & & & & $\sqrt{ }$ & $\sqrt{ }$ & $\sqrt{ }$ & $\sqrt{ }$ & $\sqrt{ }$ & & & \\
\hline & c & $\sqrt{ }$ & & & & & & & & $\sqrt{ }$ & $\sqrt{ }$ & $\sqrt{ }$ & $\sqrt{ }$ & $\sqrt{ }$ & $\sqrt{ }$ & & \\
\hline & $\mathrm{d}$ & $\sqrt{ }$ & $\sqrt{ }$ & & $\sqrt{ }$ & & & & & & $\sqrt{ }$ & $\sqrt{ }$ & $\sqrt{ }$ & $\sqrt{ }$ & $\sqrt{ }$ & & \\
\hline
\end{tabular}

\section{Conclusions}

This article incorporates preference uncertainty into coalition analysis under three solution concepts, GMR, SMR, and SEQ, in the framework of the graph model for conflict resolution. The interrelationships are investigated for the four coalition solution concepts, CNash, CGMR, CSMR, and CSEQ within each extension and across the four extensions. The proposed development is illustrated by a bulk-water export conflict occurred in Canada and structural insights are garnered about how the conflict may be 
Table 8: Coalition analysis result for CSMR and CSEQ for the Gisborne model

\begin{tabular}{|c|c|c|c|c|c|c|c|c|c|c|c|c|c|c|c|c|c|}
\hline \multirow{2}{*}{\multicolumn{2}{|c|}{ State }} & \multicolumn{7}{|c|}{$\mathrm{CSMR}_{H}$} & \multirow{2}{*}{ CSMR } & \multicolumn{7}{|c|}{$\mathrm{CSEQ}_{H}$} & \multirow{2}{*}{ CSEQ } \\
\hline & & 1 & 2 & 3 & 12 & 13 & 23 & 123 & & 1 & \begin{tabular}{|l|}
2 \\
\end{tabular} & 3 & 12 & 13 & 23 & 123 & \\
\hline & $\mathrm{a}$ & $\sqrt{ }$ & & $\sqrt{ }$ & & $\sqrt{ }$ & $\sqrt{ }$ & $\sqrt{ }$ & & $\sqrt{ }$ & & $\sqrt{ }$ & & $\sqrt{ }$ & $\sqrt{ }$ & $\sqrt{ }$ & \\
\hline \multirow{3}{*}{$s$} & $\mathrm{~b}$ & $\sqrt{ }$ & $\sqrt{ }$ & $\sqrt{ }$ & $\sqrt{ }$ & $\sqrt{ }$ & $\sqrt{ }$ & $\sqrt{ }$ & $\sqrt{ }$ & $\sqrt{ }$ & $\sqrt{ }$ & $\sqrt{ }$ & $\sqrt{ }$ & $\sqrt{ }$ & $\sqrt{ }$ & $\sqrt{ }$ & $\sqrt{ }$ \\
\hline & c & $\sqrt{ }$ & $\sqrt{ }$ & $\sqrt{ }$ & $\sqrt{ }$ & $\sqrt{ }$ & $\sqrt{ }$ & $\sqrt{ }$ & $\sqrt{ }$ & $\sqrt{ }$ & $\sqrt{ }$ & $\sqrt{ }$ & $\sqrt{ }$ & $\sqrt{ }$ & $\sqrt{ }$ & $\sqrt{ }$ & $\sqrt{ }$ \\
\hline & $\mathrm{d}$ & $\sqrt{ }$ & $\sqrt{ }$ & $\sqrt{ }$ & $\sqrt{ }$ & $\sqrt{ }$ & $\sqrt{ }$ & $\sqrt{ }$ & $\sqrt{ }$ & $\sqrt{ }$ & $\sqrt{ }$ & $\sqrt{ }$ & $\sqrt{ }$ & $\sqrt{ }$ & $\sqrt{ }$ & $\sqrt{ }$ & $\sqrt{ }$ \\
\hline & $\mathrm{a}$ & $\sqrt{ }$ & & $\sqrt{ }$ & $\sqrt{ }$ & $\sqrt{ }$ & & $\sqrt{ }$ & & $\sqrt{ }$ & & $\sqrt{ }$ & $\sqrt{ }$ & $\sqrt{ }$ & & $\sqrt{ }$ & \\
\hline & $\mathrm{b}$ & $\sqrt{ }$ & $\sqrt{ }$ & $\sqrt{ }$ & $\sqrt{ }$ & $\sqrt{ }$ & $\sqrt{ }$ & $\sqrt{ }$ & $\sqrt{ }$ & $\sqrt{ }$ & $\sqrt{ }$ & $\sqrt{ }$ & $\sqrt{ }$ & $\sqrt{ }$ & $\sqrt{ }$ & $\sqrt{ }$ & $\sqrt{ }$ \\
\hline & $\mathrm{C}$ & $\sqrt{ }$ & $\sqrt{ }$ & $\sqrt{ }$ & $\sqrt{ }$ & $\sqrt{ }$ & $\sqrt{ }$ & $\sqrt{ }$ & $\sqrt{ }$ & $\sqrt{ }$ & $\sqrt{ }$ & $\sqrt{ }$ & $\sqrt{ }$ & $\sqrt{ }$ & & $\sqrt{ }$ & \\
\hline & $\mathrm{d}$ & $\sqrt{ }$ & $\sqrt{ }$ & $\sqrt{ }$ & $\sqrt{ }$ & $\sqrt{ }$ & $\sqrt{ }$ & $\sqrt{ }$ & $\sqrt{ }$ & $\sqrt{ }$ & $\sqrt{ }$ & $\sqrt{ }$ & $\sqrt{ }$ & $\sqrt{ }$ & $\sqrt{ }$ & $\sqrt{ }$ & $\sqrt{ }$ \\
\hline & $a$ & $\sqrt{ }$ & & $\sqrt{ }$ & & $\sqrt{ }$ & & & & $\sqrt{ }$ & & $\sqrt{ }$ & & $\sqrt{ }$ & & & \\
\hline$s_{8}$ & $\mathrm{~b}$ & $\sqrt{ }$ & $\sqrt{ }$ & $\sqrt{ }$ & $\sqrt{ }$ & $\sqrt{ }$ & & & & $\sqrt{ }$ & $\sqrt{ }$ & $\sqrt{ }$ & $\sqrt{ }$ & $\sqrt{ }$ & & & \\
\hline & C & $\sqrt{ }$ & $\sqrt{ }$ & $\sqrt{ }$ & & $\sqrt{ }$ & $\sqrt{ }$ & & & 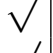 & & $\sqrt{ }$ & & $\sqrt{1}$ & & & \\
\hline & u & $\sqrt{ }$ & $\sqrt{ }$ & $\sqrt{ }$ & $V$ & $\sqrt{ }$ & $\sqrt{ }$ & & & $v$ & $\sqrt{ }$ & $v$ & $\sqrt{ }$ & $\sqrt{ }$ & & & \\
\hline
\end{tabular}

settled after considering potential coalitions.

Generally speaking, the proposed research reported herein enables an analyst to examine whether a subset of DMs can attain a better outcome by forming a coalition and coordinating their moves in a strategic conflict with three or more DMs and uncertain preference. As shown in the case study in Section 5, it can be quite tedious to carry out the coalition analysis proposed in this article. To facilitate an analyst to apply the proposed analysis, it is a worthy topic to address how to implement it into a decision support system. To facilitate computer implementation, Xu et al. [20,22] have developed an innovative matrix structure to represent moves and preferences in a graph model. It would be worthwhile to investigate how to incorporate matrix representation into this new development so that computer implementation can be conveniently tackled.

\section{References}

[1] R.J. Aumann and S. Hart (1994), Handbook of Game Theory with Economic Applications. Amsterdam: Elsevier.

[2] J. Bryant (2007), "Drama theory: dispelling the myths", Journal of the Operational Research Society 58(5): 602-613 
[3] L. Fang, K.W. Hipel, and D.M. Kilgour, Interactive Decision Making: The Graph Model for Conflict Resolution. New York: Wiley, 1993.

[4] L. Fang, K.W. Hipel, and L. Wang (2002), "Gisborne water export conflict study", Proceedings of 3rd International Conference on Water Resources Environment Research $1,432-436$.

[5] G.W. Fischer, J. Jia, and M.F. Luce (2000), "Attribute conflict and preference uncertainty: The randMAU model", Management Science 46(5): 669-684.

[6] G.W. Fischer, M.F. Luce, J. Jia (2000), "Attribute conflict and preference uncertainty: Effects on judgment time and error", Management Science 46(1): 88-103.

[7] N. Howard (1971), Paradoxes of Rationality: Theory of Metagames and Political Behavior, Cambridge, MA: MIT press.

[8] T. Inohara and K.W. Hipel (2008), "Coalition analysis in the graph model for conflict resolution", Systems Engineering 11: 343-359.

[9] T. Inohara and K.W. Hipel (2008), "Interrelationships among noncooperative and coalition stability concepts", Journal of Systems Science and Systems Engineering 17 (1): $1-29$.

[10] D.M. Kilgour and K.W. Hipel (2005), "The graph model for conflict resolution: past, present, and future", Group Decision and Negotiation 14: 441-460.

[11] D.M. Kilgour, K.W. Hipel, X. Peng, and L. Fang (2001), "Coalition analysis in group decision support", Group Decision and Negotiation 10: 159-175.

[12] M. Kitamura and T. Inohara (2011), "A new binary relation to compare viability of winning coalitions and its interrelationships to desirability relation and blockability relation", Applied Mathematics and Computation 217(13), 6176-6184.

[13] K. Kojima and T. Inohara (2012), "Coalition values derived from methods for comparison of coalition influence for games in characteristic function form", Applied Mathematics and Computation 219(3), 1345-1353. 
[14] K.W. Li, K.W. Hipel, D.M. Kilgour, and L. Fang (2004), "Preference uncertainty in the graph model for conflict resolution", IEEE Transactions on Systems, Man, Cybernetics - Part A: Systems and Humans 34 (4): 507-520.

[15] K.W. Li, D.M. Kilgour, and K.W. Hipel, "Status quo analysis of the Flathead River conflict", Water Resources Research 40(5) (2004), Art. No. W05S03 (doi: 10.1029/2003WR002596).

[16] K.W. Li, D.M. Kilgour, and K.W. Hipel (2005), "Status quo analysis in the graph model for conflict resolution", Journal of the Operational Research Society 56: 699707.

[17] K.W. Li, K.W. Hipel, D.M. Kilgour, and D.J. Noakes (2005), "Integrating uncertain preference into status quo analysis with applications to an environmental conflict", Group Decision and Negotiation 14, 461-479.

[18] J.F. Nash (1950), "Equilibrium points in n-person games", in Proc. Nat. Acad. Sci, 36: $48-49$.

[19] M.A. van Deeman (1997), Coalition Formation and Social Choice, Dordrecht: Kluwer Academic.

[20] H. Xu, K.W. Hipel, and D.M. Kilgour, "Matrix representation of solution concepts in multiple decision maker graph models", IEEE Transactions on Systems, Man, and Cybernetics-Part A 39 (1) (2009), 96-108.

[21] H. Xu, K.W. Li, K.W. Hipel, and D.M. Kilgour (2009), "A matrix approach to status quo analysis in the graph model for conflict resolution", Applied Mathematics and Computation 212: 470-480.

[22] H. Xu, D.M. Kilgour, and K.W. Hipel (2010), "Matrix representation and extension of coalition analysis in group decision support", Computers and Mathematics with Applications, 60: 1164-1176. 